
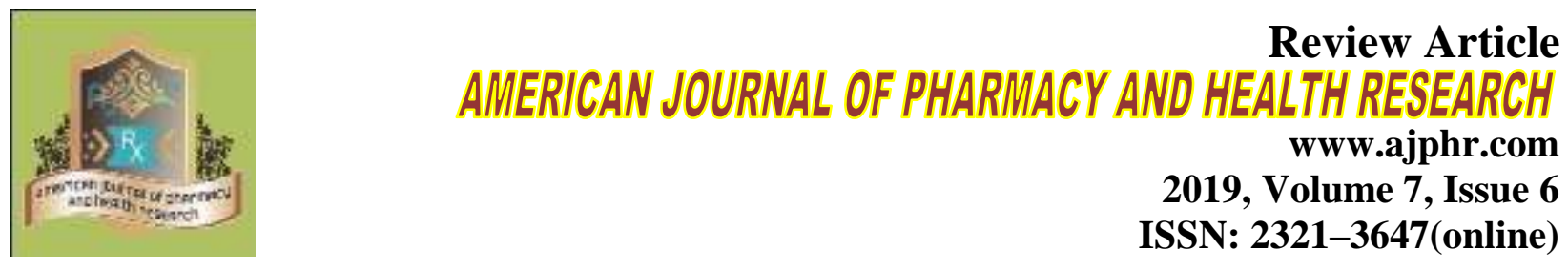

\title{
Antipsychotic drug induced sexual dysfunction
}

Prashant Mathur ${ }^{1}$, Sandeep Uniyal

\begin{abstract}
Sexual dysfunction is defined as any discomfort experienced by an individual or a couple during any stage of a normal sexual activity. According to ICD-10 sexual dysfunction is a condition in which an individual or couple is unable to perform sexual intercourse as both would wish. Atherosclerosis of the arteries supplying genital tissue which elevate sexual function. Typical antipsychotic induce relatively high chances of developing sexual dysfunction in comparison to atypical antipsychotics.
\end{abstract}

Keywords : Sexual dysfunction, Antipsychotics, Sexual intercource, ICD-10. 


\section{INTRODUCTION}

Sexual dysfunction is defined as any discomfort experienced by an individual or a couple during any stage of a normal sexual activity. Even sexual dysfunction occur both men and women. Sexual relationship is an important issue and sexual dysfunction has a great impact on population and their quality of life ${ }^{(1)}$. According to ICD-10 sexual dysfunction is a condition in which an individual or couple is unable to perform sexual intercourse as both would wish. The main cause of this may lack of interest, lack of enjoyment and other physiological response failure or inability to control orgasm ${ }^{(2)}$. Atherosclerosis of the arteries supplying genital tissue which elevate sexual function. These are the conditions which increase sexual problems are Hyperprolactinaemia, hypertension, obesity ${ }^{(1,2)}$.

Healthy physical relationship between a couple plays an important role in marital life. if both are not satisfied with each other then life became unromantic or incomplete. Sexual intercourse is vital link between husband and wife. During sexual activity sometime individual privation of a required sexual intercourse ${ }^{(3)}$. Antipsychotic drug induced sexual dysfunction is a major problem associated with the treatment of mental disease like hypertension, depression, schizophrenia, and diabetes. Major depressive patient may also develop psychotic feature like hallucination or delusion and the symptoms of major depression include (concentration difficulty, irritability, social isolation, loss of appetite, threats of suicide) these symptoms affect daily life of patient. it is important for the patient to report all the adverse effect or unwanted effect of the prescribed medication which may affect quality of life like decreased libido (in both man and women), erectile dysfunction (in men) .prescriber should be aware for the drug therapy because sexual problem are sometimes difficult for the patient to share about actual problem for example delayed orgasm or decreased libido ${ }^{(4)}$. Arizona sexual experience scale (ASEX) is data collection tools were demographic data questionnaire including age gender, level of education, type of drug received, type of mental and physical disease and hospitalization word ${ }^{(3)}$.

Types of sexual dysfunction: -

- Sexual desire disorder - decreased libido (absence of sexual desire in both male and female.

- Sexual arousal disorder - arbitrate by CNS and parasympathetic system.

- Sexual pain disorder -lack of insufficient lubrication of vagina during sex.

- Orgasm disorder- failure to achieve climax. Delayed ejaculation.

\section{Sexual desire}


Desire is believed to be a combination of cognitive, physiologic, and developmental factors. Androgens may play a part in sexual desire in men and women; however, the mechanism is unclear. Variations in testosterone concentrations have been correlated with the extent of sexual desire, and administration of exogenous testosterone in men has been found to increase sexual thoughts and desire. Other hormones, such as estrogen and prolactin, may also contribute to dysfunction. Elevated prolactin secondary to reduced central dopaminergic activity can decrease sexual interest. Hormonal changes during the menstrual cycle has been considered as a primary component of desire in women; however, inconsistent results in the importance $(6,8,9)$

Typical antipsychotics such as Fluphenazine, Haloperidol, Chlorpromazinc, Thioridazine, and Thiothixene can all decrease sexual desire. The antidopaminergic action of the antipsychotics increase prolactin concentrations thereby decreasing libido. The atypical agent Risperidone also effects prolactin levels and is believed to affect sexual functioning in a similar manner. Olanzapine, Clozapine, and Quetiapine are all associated with minimal effects on prolactin and should be considered as alternatives. Antidepressants can also decrease desire, possibly due to their anticholinergic and sedative properties. Problems have been reported with several tricycle antidepressants (TCAs), including Amitriptyline, Imipramine, Desipramine and Nortriptyline, the Monoamine oxidase inhibitors (MAOIs) Phenelzine, Isoearboxazid and tranylcypromine, Amoxapine, and Trazodone ${ }^{(9,10)}$.

\section{Impotence or Erectile Dysfunction}

Impotence is defined as the inability to achieve or to maintain erection sufficient to allow satisfactory sexual intercourse. Disease states such as diabetes, high blood pressure, and cardiac dysfunction are associated with impotence and should be considered as contributing factors. The etiology of impotence may be hormonal, neurologic, vascular, and/or psychogenic ${ }^{(5,6,7)}$. With vascular disease being the single mom's common cause. Arousal has the physical characteristics of penile tumescence in the male and pelvic vasocongestion and vagina lubrication in the female. The mechanism of the penile tumescence is thought to be controlled by two pathways: the parasympathetic pathway controlling the reflexogenic erections and the sympathetic pathway

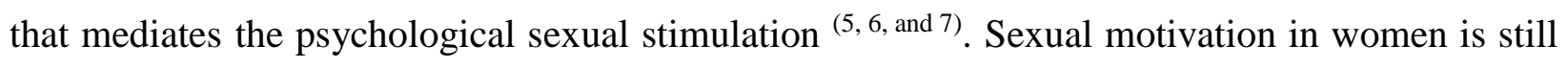
under research. Female arousal is thought to be mediated by a peptidergic innerration involving a vasoactive intestinal peptide. Adrenergic and cholinergic nerves have been suggested to play a part in erectile dysfunction. The physiology of erection begins with a parasympathetic neural impulse, which is transmitted to the arterioles within the corpus cavernosum via the pelvic plexus. The nonadrenergic neurotransmitter nitric oxide induces relaxation of the cavernosal 
tissue allowing blood flow to the penis ${ }^{(8)}$. As pressure within the corporeal bodies increases, the small emissary veins become occluded, decreasing venous outflow and trapping blood within the corpus cavemosum. Erection is maintained until a sympathetic neural impulse from the hypogastric plexus resuits in ejaculation, and parasympathetic tone is reduced, causing detumescence $^{(9,10)}$. Antidepressants that contribute to erectile dysfunction include the TCAs, especially the tertiary amines, and the MAOIs Amoxapine and Trazodone. The mechanism by which antidepressants interfere with erection is unclear. Antipsychotics associated with erectile dysfunction include Fluphenazine, Chlorpromazine, Haloperidol, Pimozide, and Thioridazine ${ }^{(6)}$. Central and peripheral action of these drugs on dopaminergic blockade has been implicated as the causative factor in erectile dysfunction ${ }^{(11)}$.

Antihypertensive agents are also associated with erectile dysfunction also other drug like Hydrochlorothiazide, Chlorthalidone, and high-dose Spironolactone have all been implicated ${ }^{(12)}$. Sympatholytic agents such as Methytdopa, Guanethidine, and Reserpine not only affect libido but also erectile function. Lipophilic beta blockers (e.g., Propranolol) are more likely to cause dysfunction than the hydrophilic beta blockers. Reports of dysfunction with pindolol, metoprolol, and nadolol are less common. Angiotensin-converting enzyme inhibitors such as Captopril and Enalapril have minimal sexual side effects and should be considered as alternative therapy. Other medications that may cause erectile dysfunction include lithium, digoxin, Alprazolam and Clonidine are medications that effecting erectile dysfunction.

\section{Premature Ejaculation}

Premature or rapid ejaculation is defined as a persistent or recurrent ejaculation with minimal sexual stimulation before, on, or shortly after penetration and before the individual wishes it. The pathophysiology of rapid ejaculation is unknown; however, many believe it to be psychologically based. Conflicts with the sexual partner, hostility toward women, performance anxiety, and organic factors such as prostatitis, nrethritis, and pelvic fracture have all induced premature ejaculation ${ }^{(11)}$. Drug withdrawal from drugs such as Trifluoperazinc and narcotic agents has also caused this problem.

\section{Orgasm}

The mechanism by which the orgasmic stage of sexual intercourse is produced in men includes two phases: an emission phase and an ejaculatory phase. The emission phase, under sympathetic control, is mediated by norepinephrine, which acts on alpha-adrenergic receptors, the vas deferens, the prostate, and the seminal vessels, which help to promote the flow of the seminal fluid into the urethra ${ }^{(6,7)}$. The ejaculatory phase is mediated by a sacral spinal reflex, which 
involves the striated muscles around the bulbar urethra, that contract and causes the projection of semen. Little information exists regarding the physiology of female orgasm. Oxytoein has been the subject of study, but before a definite relationship can be established, further study is needed. Central increases in serotonergic receptor activation have been associated with orgasmic inhibition or anorgasmia. Antipsychotics are believed to contribute to orgasmic dysfunction by their alpha-adrenergic blocking effects. Those that have been associated with orgasmic or ejaculatory disturbances include Trifluoperazine, Perphenazine, Mesoridazine, Chlorprothixene, Chlorpromazine, and Thioridazine, with Thioridazine causing the most problems. Fluphenazine and haloperidol have also been shown to cause these disturbances. Antidepressants affecting orgasm and ejaculation include SSRIs such as Fluoxetine, Paroxetine, and Sertraline; MAOIs such as Phenelzine and Tranylcypromine; TCAs such as Desipramine, Imipramine, Nortriptyline, Amitriptyline, Elomipramine, and Doxepin; and Amoxapine and Trazodone. Benzodiazepines such as Alprazolam and lorazepam have been shown to cause an ejaculatory delay ${ }^{(6)}$. The mechanism of this is unclear but may be related to the sedative properties of the benzodiazepines. Other agents that may cause problems with orgasm and ejaculation include Guanethidine, Labetalol, Methyldopa, Reserpine, and Nifedipine.

\section{Hyperprolactinaemia and its role in sexual dysfunction}

Hyperprolactinaemia is main cause of sexual dysfunction high level of prolectin hormone secreted from lactotroph cells in the anterior pituitary gland due to the dopamine which may inhibit the effect on prolectin secretion. ${ }^{(5,6)}$. Reduction in dopamine can occur via the administration of antipsychotics which act on D2 receptor in the tuberoinfundibular pathway of the brain reduced amount of dopaminergic input to the lactotroph cells results in a sudden increase in prolectin secretion. high serum prolectin level have effect on sexual dysfunction, decrease libido, reproductive health, hypogonadism, infertility in women and low sperm count in men and amenorrhea ${ }^{(5,6)}$.

\section{Pathophysiology of antipsychotics}

Antipsychotic drug mechanisms which can cause sexual dysfunction are histamine receptor antagonism, dopamine receptor antagonism, cholinergic receptor antagonism alpha-adenergic alpha receptor antagonism and dopamine D2 receptor antagonism. With combine to histaminergic receptors may spoil arousal by increasing sedation activity. Dopaminergic receptor antagonism may decrease the libido by interfere drive .Blockage of dopamine D2 receptor in the tuberoinfundibular pathway by antipsychotics decrease libido, erectile dysfunction and irregular orgasm. Cholinergic receptor antagonism may increase erectile dysfunction by decreasing 
peripheral vasodilatation, alpha adrenergic alpha receptor antagonism can decrease peripheral vasodialation causing erectile dysfunction in men and decrease lubrication in women .irregular

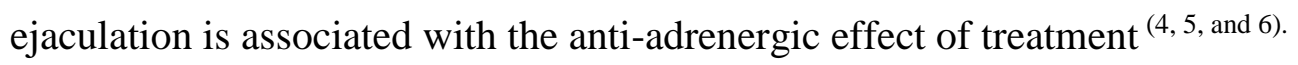

The main cause of sexual dysfunction is Hyperprolactinaemia in male and female using antipsychotics by anterior mechanism. Due to the blockage of dopamine D2 receptor Risperidone, Haloperidol, are prolectin elevating antipsychotics. (4)

\section{Sexual dysfunction due to typical and atypical antipsychotic drug.}

According to study by Hummer and his colleagues correlate sexual dysfunction among schizophrenies on Clozapine an atypical antipsychotic with those on haloperidol a typical antipsychotic. 100 cases on Clozapine and 53 cases on haloperidol including both genders were rated in UKU side effect rating scale. 58 percent had decrease libido, 27 percent impaired arousal and 22 percent orgasm disorder in the haloperidol group and on other hand 50 percent decrease sexual desire, 24 percent arousal problems and 21 percent orgasm disorder (delayed ejaculation) in the Clozapine group. Thus Clozapine and haloperidol with regard to in their tendency to induce sexual dysfunction.

\section{Drug induced sexual dysfunction}

\section{Haloperidol}

Haloperidol is a butyrophenone-derivative which inhibit the ascending reticular system via the caudate nucleus it blocks postsynaptic dopamine receptor in the mesolimbic dopaminergic system so increase turnover of brain dopamine. It acts on D2-receptor and show effect on 5HT2 and $@_{1}$ receptors. Haloperidol widely used to treat delirium because it shows decrease hypotension, sedation and also has anticolinergic effect in comparison of other antipsychotic ${ }^{(15)}$. It is high potency first generation neuroleptic agent ${ }^{(16)}$. It raises serum prolactin levels to $20-40$ $\mathrm{ng} / \mathrm{ml}$ in therapeutic doses and it may cause libido, amenorrhea, gynecomastia, erectile dysfunction/ sexual dysfunction, and galactorrhea.

\section{Risperidone}

Risperidone is a second generation antipsychotic agent used for acute treatment of schizophrenia and psychotic disorder like bipolar disorder and mania. It is a potent inhibitor of serotonin 5- $\mathrm{HT}_{2}$ and dopamine $\mathrm{D}_{2}$ receptor it undergoes a- hydroxylation in the liver predominantly catalyzed by the cytochrome $\mathrm{P}_{450}$ enzyme, CYP2D6 TO produce a-hydroxy-Risperidone metabolic route for Risperidone include oxidative N-dealkylation and hydroxylation to 7-hydroxy-risperdone ${ }^{(17)}$. Risperidone is a representative atypical antipsychotic drug with a high probability of prolactin elevation. Risperidone elevates the serum prolactin in a dose dependent manner and up to a level 
of 30 60 ng/ml when used at therapeutic doses. A small scale study reported gynecomastia, galactorrhea, and priapism in male patients. Further, female patients were reported to experience amenorrhea and galactorrhea. ${ }^{(4)}$

\section{Clozapine}

Clozapine is an atypical antipsychotic which is benzodiazepine and have central antidopaminergic activity peak blood level occur in body is 2.1 hours and mean half life of 12 hour and also $95 \%$ bound to plasma and completely metabolized prior to excretion ${ }^{(18)}$. Clozapine is a prototypical atypical antipsychotic whose metabolism covariant with $\mathrm{CYP}_{1} \mathrm{~A}_{2}$ activity ${ }^{(19)}$. Clozapine also induce a risk of agranulocytosis an increased reduction in the number of white blood cell in the body. The inability of Clozapine to elevate serum prolectin level may be related to the activate the effect of Clozapine on tuberoinfundibular dopamine neurons and to achieve effective blockade of dopamine $\mathrm{D}_{2}$ receptor of pituitary. It also exerts antagonism of $\mathrm{D}_{1}$ receptor and show interaction of Clozapine among $\mathrm{D}_{2}, \mathrm{D} 1, \mathrm{D} 4$ and $5 \mathrm{HT}_{2}$ receptors produced in a characteristic conversion in function of pre and post synaptic dopamine elements ${ }^{(20)}$.

Clozapine was associated with low prolactin serum level as compared to classical antipsychotics Clozapine $27 \mathrm{ng} / \mathrm{ml}$. Clozapine produces a low incidence of Hyperprolactinaemia related adverse Sexual events including decreased libido and impaired arousal relative to classical antipsychotics (3).

\section{Olanzapine}

Olanzapine elevates prolactin level increase level of prolactin may abolish hypothalamic $\mathrm{GnRH}$ and this promote in reduced pituitary gonadotropin secretion and it may inhibit reproduction function by damaged gonadal steroidogenesis in both gender patients gynecomastia(men boobs), galactorrhea, amenorrhea and erectile dysfunction have been reported in patient taking Olanzapine $^{(21)}$.

Olanzapine temporarily increases the prolactin level. Moreover, in most cases, the elevated prolactin level returns to a normal level. In a double blind clinical trial, prolectin elevation Olanzapine produces a low incidence of adverse sexual events in men as compared to

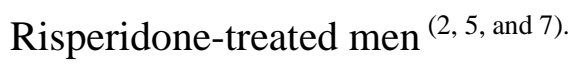

Olanzapine is second generation antipsychotic agent and bind with serotonin $5 \mathrm{HT}{ }_{2} \mathrm{~A} /{ }_{2} \mathrm{C}$, dopamine $\mathrm{D}_{1-4}$, histamine $\mathrm{H}_{1}$ and adrenergic receptor. It is an antagonist with balanced attraction binding for serotonin in $5 \mathrm{HT}_{3}$ and muscarinic $\mathrm{M}_{1-5}$ and weakly bind with GABA A, B-adrenergic and $\mathrm{B}_{2} \mathrm{D}$ receptor ${ }^{(21)}$. 


\section{Aripiprazole}

Aripiprazole used for the treatment of schizophrenia and bipolar disorder it is a dopamine partial agonist and also has binding attraction for the serotonin $5 \mathrm{HT}_{2} \mathrm{~A}$ receptor. Aripiprazole reduce dopaminergic neurotransmission through $\mathrm{D}_{2}$ partial agonism it bind with CNS receptor mainly partial $\mathrm{D}_{2}$ agonist, $5 \mathrm{HT}_{2} \mathrm{~A}$ antagonist, and partial $5 \mathrm{HT}_{1} \mathrm{~A}$ agonist. It has lower intrinsic efficacy that's why it triggers lower than dopamine and higher than an antagonist it modulates neurotransmission mainly mesolimbic and mesocortical pathway via partial D2 agonist (22).

According to double-blind clinical trial studies, Aripiprazole did not increase the prolactin level or normalize prolactin levels in patients with elevated baseline levels caused by typical antipsychotics. Aripiprazole was associated with a significant improvement in sexual desire, erectile dysfunction, and ejaculatory disorder ${ }^{(3,5)}$.

\section{MANAGEMENT OF ANTIPSYCHOTICINDUCED SEXUAL DYSFUNCTION}

Dopamine agonist (Bromocriptine, Cabergoline) and dopamine releasing agent (Amantadine) were tried in a patient suffering from sexual dysfunction secondary to antipsychotics, The patients reported significant improvement while taking Sildenafil in the number of adequate $\operatorname{erections}^{(3)}$.

\section{CONCLUSION}

After reviewing several articles on antipsychotic drug induced sexual dysfunction it is identified that atypical antipsychotic drug induced sexual dysfunction especially erectile dysfunction in man and decreased libido in both men \& women. So it is responsibility for a psychiatrist the choice of antipsychotic is appropriate for minimum the risk of sexual dysfunctions.

\section{REFERENCE}

1. Douma stella ; Doumas michale, tsokiris alexandros, zomboulis chrysonthos . at el .male and female sexual dysfunction ; is hypertension an innocent by stander or a major contributer, Rev bras hipertenss vol 14(3); 139-49.2007.

2. Campbell MM, stein DJ sexual dysfunction; A sexual review of south African research department of psychiatry and mental health, faculty of health science, university of cape town, south Africa. June 7 . 2014; 104(6) 440-4.

3. Suzanne E. Fecik, pharmd psychopharmacy research and education program, "western missouri mental health center, university of missouri-kansas city, kansas city, missouri, psychiatrists $3 ; 6 ; 176-181,1998$. 
4. Ahmadzadeh G, Shahin A. Sexual dysfunctions in the patients hospitalized in psychiatric wards compared to other specialized wards in Isfahan, Iran, in 2012. Adv Biomed Res $2015 ; 4: 225$.

5. Yeon Won Park, Yooseok Kim, Jun Ho Lee department of urology , national police hospital, seole, korea Antipsychotic-Induced Sexual Dysfunction and Its Management 2012.30.3.153.

6. Bains S, Shah AA (2012) Sexual Side Effects of Antipsychotic Drugs. Adv Pharmacoepidem Drug Safety 1:109. Dept. of Psychiatry, Family and Community medicine Baylor College of medicine, USA.

7. Schiavi RC, Segraves RT. The biology of sexual function. psychiat clin north AM $1995 ; 18 ; 7-23$

8. Segraves RT .hormones and libido.in; labium SR rosen RC eds. Sexual desire. Disorder new York guiford press 1998;27

9. Davidson JM Kwan M. Green leaf wj. Hormonal replacement and sexuality clin endocrinol metals 1982 11; 599.

10. Smith PJ, Talbert RL. Sexual dysfunction ant antihypertensive and antipsychotic agent .clin pharm 1986;5;373-84

11. Segraves RT. drug and desire. In Leiblum SR , Rosen RC Eds, sexual desire disorder new York ,guitord press 1988;313-47

12. NIH Consensus development conference statement NIH; impotence Int J Impot Res $1993 ; 5 ; 181-99$

13. Althof SE, seftel AD. The evaluation and management of erectile dysfunction .Psychiat clin north AM 1995;18-92

14. Nagaraj AK, Pai NB, Nanjegowda RB, Rajagopal R, Shivarudrappa NK, Siddika N. Etiology and management of sexual dysfunction. Online Journal of Health and Allied Sciences. 2009; 8(2):1.

15. Miller A, McKee A, Mazer CD. Sedation, Analgesia, and Related Topics. In Cardiothoracic Critical Care 2007 (pp. 53-70).S

16. Grossman M, editor. Reading Renaissance Ethics. Routledge; 2007 Mar 12.

17. Germann D, Kurylo N, Han F. Risperidone. In Profiles of Drug Substances, Excipients and Related Methodology 2012 Jan 1 (Vol. 37, pp. 313-361). Academic Press.

18. Seksel K. Behavior-modifying drugs. In Small animal clinical pharmacology 2008 (pp. 126-147). Saunders Elsevier, St Louis (MO). 
19. Flockhart DA, Desta Z. Pharmacogenetics of drug metabolism. In Clinical and Translational Science 2009 (pp. 301-317).

20. Meltzer HY, Gudelsky GA. Dopaminergic and serotonergic effects of Clozapine. Implications for a unique clinical profile. Arzneimittel-Forschung. 1992 Feb; 42(2A):268-72.

21. Guzman F. Use of Second-Generation Antipsychotics in Bipolar.2018 Jan

22. Citrome L. A review of Aripiprazole in the treatment of patients with schizophrenia or bipolar I disorder. Neuropsychiatric disease and treatment. 2006 Dec; 2(4):427.

AJPHR is

Peer-reviewed

monthly

Rapid publication

Submit your next manuscript at

editor@ajphr.com / editor.ajphr@gmail.com 